\title{
Impact of the COVID-19 lockdown on the arrhythmic burden of patients with implantable cardioverter-defibrillators
}

\author{
Biagio Sassone MD ${ }^{1,2,3}$ (1) ～～Santo Virzì MD ${ }^{2} \quad$ Matteo Bertini MD, $\mathrm{PhD}^{4}$ | \\ Giovanni Pasanisi MD ${ }^{3}$ ～Lamberto Manzoli MD, MPH ${ }^{5}$ ～Jonathan Myers MD ${ }^{6,7}$ \\ Giovanni Grazzi MD ${ }^{8,9}$ | Daniele Muser MD, $\mathrm{PhD}^{10,11}$ [
}

${ }^{1}$ Department of Translational Medicine, University of Ferrara, Ferrara, Italy

${ }^{2}$ Department of Emergency, Division of Cardiology, SS.ma Annunziata Hospital, Ferrara, Italy

${ }^{3}$ Department of Emergency, Division of Cardiology, Delta Hospital, Ferrara, Italy

${ }^{4}$ Cardiological Centre, Department of Translational Medicine, University of Ferrara, Ferrara, Italy

${ }^{5}$ Department of Medical Sciences, University of Ferrara, Ferrara, Italy

${ }^{6}$ Division of Cardiology, VA Palo Alto, Palo Alto, California, USA

${ }^{7}$ Stanford University School of Medicine, Stanford, California, USA

${ }^{8}$ Centre for Exercise Science and Sport, University of Ferrara, Ferrara, Italy

${ }^{9}$ Healthy Living for Pandemic Event Protection (HL-PIVOT) Network, Chicago, Illinois, USA

${ }^{10}$ Cardiac Electrophysiology, Cardiovascular Division, Hospital of the University of Pennsylvania, Philadelphia, Pennsylvania, USA

${ }^{11}$ Cardiothoracic Department, Udine Civil Hospital, Udine, Italy

\section{Correspondence}

Prof. Biagio Sassone, MD, Cardiology Division, SS.ma Annunziata Hospital, via Giovanni Vicini 2, 44042 Cento, Ferrara, Italy.

Email:biagio.sassone@gmail.com

\begin{abstract}
Background: In Italy, a nationwide full lockdown was declared between March and May 2020 to hinder the novel coronavirus disease 2019 (COVID-19) pandemic. The potential individual health effects of long-term isolation are largely unknown. The current study investigated the arrhythmic consequences of the COVID-19 lockdown in patients with defibrillators (ICDs) living in the province of Ferrara, Italy.

Methods: Both the arrhythmias and the delivered ICD therapies as notified by the devices were prospectively collected during the lockdown period (P1) and compared to those occurred during the 10 weeks before the lockdown began (P2) and during the same period in 2019 (P3). Changes in outcome over the three study periods were evaluated for significance using McNemar's test.

Results: A total of 413 patients were included in the analysis. No differences were found concerning either arrhythmias or shocks or anti-tachycardia pacing. Only the number of patients experiencing non-sustained ventricular tachycardias (NSVTs) during $\mathrm{P} 1$ significantly decreased as compared to $\mathrm{P} 2(p=0.026)$ and $\mathrm{P} 3(p=0.009)$. The subgroup analysis showed a significant decrease in NSVTs during P1 for men (vs. P2, $p=0.014$; vs. $\mathrm{P} 3, p=0.040$ ) and younger patients (vs. P2, $p=0.002$; vs. $\mathrm{P} 3, p=0.040$ ) and for ischemic etiology (vs. P2, $p=0.003$ ). No arrhythmic deaths occurred during P1. Conclusions: The complete nationwide lockdown, as declared by the Italian government during the first COVID-19 pandemic peak, did not impact on the incidence of arrhythmias in an urban cohort of patients with ICDs.
\end{abstract}

KEYWORDS

arrhythmia, COVID-19, defibrillator, lockdown

\section{1 | INTRODUCTION}

Abbreviations: ATP, anti-tachycardia pacing; COVID-19, coronavirus disease 2019; ICD, implantable cardioverter-defibrillator; NSVT, non-sustained ventricular tachycardia; P1, study period 1: the lockdown period (March 9th and May 18th, 2020); P2, study period 2: 10 weeks before the lockdown began; P3, study period 3: the corresponding period in 2019 (March 9th and May 18th, 2019); SVA, supraventricular arrhythmia; VT, ventricular tachycardia
The first cases of severe acute respiratory syndrome-coronavirus-2 (SARS-CoV-2) infection were reported in late 2019 in China and the novel coronavirus disease (COVID-19) began to take on the characteristics of a pandemic beginning in March 2020. Italy was the

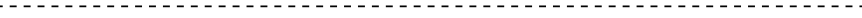
This is an open access article under the terms of the Creative Commons Attribution-NonCommercial-NoDerivs License, which permits use and distribution in any medium, provided the original work is properly cited, the use is non-commercial and no modifications or adaptations are made.

(C) 2021 The Authors. Pacing and Clinical Electrophysiology published by Wiley Periodicals LLC. 
first European country overwhelmed by COVID-19. Since COVID-19 was spreading rapidly in late February 2020, the Italian government declared a nationwide lockdown between March 9th and May 18th, 2020 in efforts to contain human-to-human virus transmission and to prevent a collapse of the health care system. The enforced restrictive social measures and the mandatory in-home confinement for most Italian residents raised concerns about the ability to maintain wellestablished favorable effects of a healthy lifestyle in patients with cardiovascular disease.

While COVID-19 has been frequently associated with a high incidence of both atrial and ventricular arrhythmias, ${ }^{1,2}$ the potential effects of long-term isolation on the incidence of cardiac arrhythmias are largely unknown.

Implantable cardioverter-defibrillators (ICDs) represent a unique tool for continuous monitoring of cardiac rhythm in patients at high risk for sudden cardiac death. The current multicenter study investigated the arrhythmic consequences of the lockdown in a cohort of patients with ICDs in Ferrara, a northern Italian urban province that has been heavily affected by the COVID-19 pandemic.

\section{2 | METHODS}

We performed a multicenter cohort study including all patients aged $>18$ years who underwent ICD implantation for primary or secondary prevention of sudden death before March 9th, 2019 and who were still alive on March 9th, 2020. The routine use of remote monitoring of cardiac implantable electronic devices has been strongly recommended in the context of the COVID-19 pandemic, in order to avoid at the most the in-person visits at the device referral centers. ${ }^{3}$ Accordingly, all patients were required to have remote follow-up by homemonitoring technology at the cardiology centers of the public hospitals of Ferrara. Ferrara is a northern Italian province of the Emilia Romagna region, one of the areas most affected by the novel coronavirus pandemic. Both the arrhythmic events and the delivered ICD therapies as notified by the device were prospectively collected during the lockdown (study period 1, P1) and compared to those occurred during the 10 weeks before the lockdown began (study period 2, P2) and during the corresponding period across March-May 2019 (study period 3, P3). Accordingly, patients who underwent ICD implantation after March 9th, 2019 were excluded from the analysis. We compared P1 initially with P2 to investigate any abrupt change in arrhythmic event rate, then with P3 to determine any effect related to the wellknown seasonal fluctuation of arrhythmic risk. The three aforementioned study periods were compared for the following ICD event notifications: ventricular tachycardias (VT) of $\geq 3$ consecutive ventricular complexes at rate $>100 \mathrm{bpm}$ terminating spontaneously, detected and stored as "monitor" events; shock therapy or anti-tachycardia pacing (ATP) delivered appropriately - according to device programming - for terminating ventricular arrhythmias; inappropriate shocks delivered for cardiac rhythm-related problems (e.g. atrial fibrillation); and new episodes of sustained (> $30 \mathrm{~s}$ ) supraventricular arrhythmias. Further, untreated (i.e., monitor) VTs were divided into non-sustained (NSVT)
TAB LE 1 Demographic and clinical characteristics of the study patients $(n=413)$

\begin{tabular}{|ll|}
\hline Variables & Mean \pm SD or $n(\%)$ \\
\hline Age, years & $70 \pm 13$ \\
\hline Male & $313(76)$ \\
\hline Ischemic etiology & $201(49)$ \\
\hline NYHA functional class & \\
\hline I & $191(46)$ \\
\hline II & $181(44)$ \\
\hline III & $36(9)$ \\
\hline IV & $5(1)$ \\
\hline History of atrial fibrillation & $123(30)$ \\
\hline Left ventricular ejection & $37 \pm 12$ \\
\hline fraction, \% & \\
\hline Device type & $247(60)$ \\
\hline ICD & $166(40)$ \\
\hline CRT-D & \\
\hline
\end{tabular}

CRT-D, cardiac resynchronization therapy with defibrillator back-up; ICD, implantable.

cardioverter-defibrillator; NYHA, New York Heart Association.

or sustained VT, defined as lasting $\leq 30 \mathrm{~s}$ or $>30 \mathrm{~s}$, respectively. Any death occurring during the lockdown was also recorded. Patients who were diagnosed with COVID-19 by nasopharyngeal swab during P1 were excluded from the analysis. This study was approved by the local Internal Review Board and involves three hospitals in the province of Ferrara, Italy.

Statistical analyses were performed using Stata 13.1 (Stata Corp., College Station, TX, 2014). Changes in outcomes throughout the three study periods were evaluated for significance using McNemar's test. Significance was defined as a two-tailed test with $p<0.05$ for all analyses.

\section{3 | RESULTS}

A total of 414 patients who underwent ICD implantation before March 9th, 2019 and who were still alive on March 9th, 2020 were included. Of the initial study population, one patient was excluded from the analysis owing to a definitive diagnosis of COVID-19. Demographic and clinical characteristics of patients are shown in Table 1. Briefly, patients had a mean age of $70 \pm 13$ years and $76 \%$ were male; the etiology of cardiomyopathy was ischemic in $49 \%$ of patients and $40 \%$ of ICDs had cardiac resynchronization therapy capability. Medical therapy, including $\beta$-blockers, angiotensin-converting enzyme inhibitors or angiotensin receptor blockers, loop diuretics and antialdosteronics, did not differ during the analysis periods (Table 2).

During P1 the number of patients showing at least one notified arrhythmic event by the device was mildly lower than during P2 and P3 ( $n=66$ [16\%], $n=83$ [20\%] and $n=81[20 \%]$, respectively). As shown in Figure 1, no differences were found in the prevalence of reported ventricular arrhythmias, except for the number of patients 
TAB LE 2 Medication therapy of the 413 study patients in each of the three selected periods

\begin{tabular}{|c|c|c|c|}
\hline \multirow[b]{2}{*}{ Drug } & \multicolumn{3}{|c|}{ Number of patients (\%) } \\
\hline & Mar-May 2019 & Dec 2019 - Feb 2020 & $\begin{array}{l}\text { Mar-May } \\
2020\end{array}$ \\
\hline ACEIs/ARBs & $321(78)$ & $333(81)$ & $325(79)$ \\
\hline Loop diuretics & $291(70)$ & $283(69)$ & $279(68)$ \\
\hline
\end{tabular}

Medication therapy, for each indicated drug group, resulted not significantly different during the three selected periods as all p-values were $>0.05$ and they have not been shown to avoid redundancy.

ACEls, angiotensin-converting enzyme inhibitors; ARBs, angiotensin receptor blockers; Dec, December; Feb, February; Mar, March; MRAs, mineralocorticoid receptor antagonists.

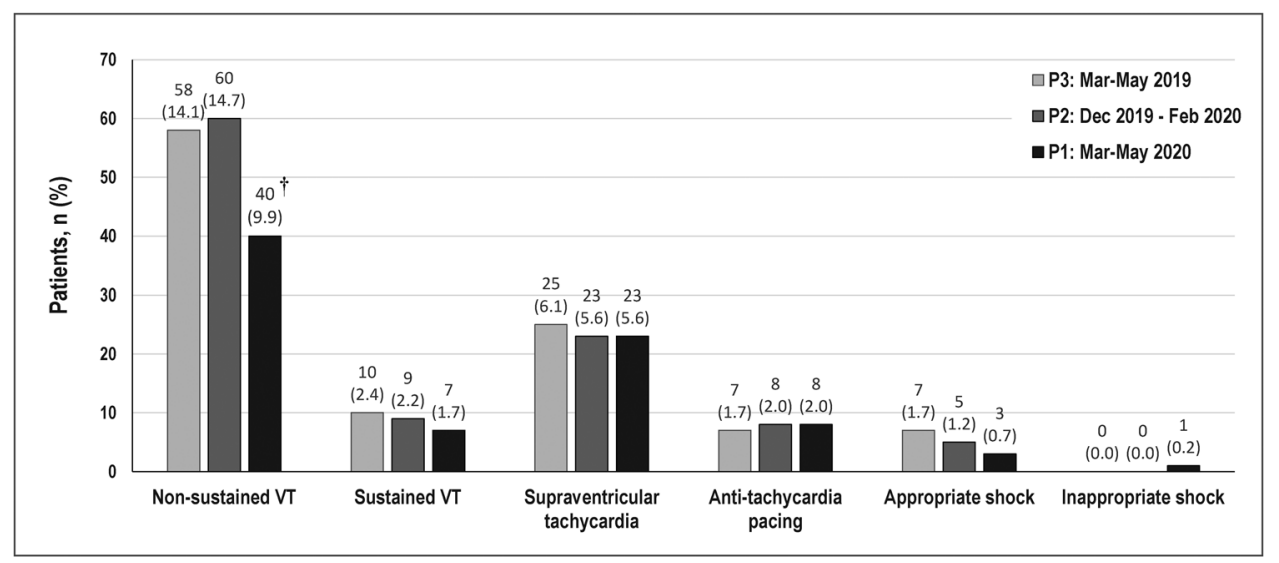

FIGURE 1 Events notified by implantable cardioverter-defibrillators in each of the three selected study periods. Non-sustained VT refers to detected, untreated and stored ventricular tachycardia lasting $\leq 30 \mathrm{~s}$. Sustained VT refers to detected, spontaneously terminated and stored ventricular tachycardia lasting $>30 \mathrm{~s} .{ }^{\dagger}$ McNemar's test: $\mathrm{P} 1$ versus $\mathrm{P} 3, p=0.026$ and $\mathrm{P} 1$ versus $\mathrm{P} 2, p=0.009$. All other $p$-values were not significant (> 0.05), and they have not been shown to avoid redundancy. Dec, December; Feb, February; Mar, March; VT, ventricular tachycardia

experiencing NSVTs, which decreased by roughly $33 \%$ as compared to both P2 ( $p=0.009)$ and P3 $(p=0.026)$. Subgroup analysis showed a significant decrease in NSVTs during P1 for men (vs. P2, $p=0.014$; vs. P3, $p=0.040$ ) and younger patients ( $<75$ years, vs. P2, $p=0.002$; vs. P3, $p=0.041$ ) and for ischemic etiology (vs. P2, $p=0.003$ ) (Table 3). We also found no significant difference concerning the incidence of SVAs during the lockdown period (5.6\%) compared to the same period of the previous year (6.1\%) or the same amount of time immediately preceding the lockdown (5.6\%). Our results have not changed even after including the only episode of inappropriate shocks delivered for new-onset atrial fibrillation that occurred during the lockdown period. Three patients died during the lockdown; the deceased patients experienced neither arrhythmic nor COVID-19-related deaths.

\section{4 | DISCUSSION}

A growing number of governments across the world have mandated various forms of isolation in efforts to hinder the spread of the COVID19 pandemic. In early March 2020, the Italian government declared the total lockdown to be applied to the whole national territory, then to the province of Ferrara as well. Several compulsory restrictions were applied indiscriminately to the general population for 69 days, with a hard impact on the social and economic country life. According to the definitions proposed by Haider et al. ${ }^{4}$ the Italian lockdown consisted of four main interventions: firstly, the geographic containment across the entire country, banning any non-essential movements in and out of the municipality of residence, with exceptions only for necessary commodities (e.g., the flow of food) and employments (e.g., medical assistance, public security); secondly, the forced in-home confinement, halting any outdoor activities except those essential (e.g., shopping for food supply); thirdly, the absolute prohibition of crowding and gatherings of people, including funerals and sporting events at any level; fourthly, closure of schools and universities, religious venues, shops and premises, with rare exemptions to be applied to essential businesses and strategic industries (e.g. pharma and food processing). Our data showed no increase of both atrial and ventricular arrhythmias or delivered ICD therapies during the lockdown period as compared to either the 10week period before the lockdown began or the corresponding period in 2019. 
TAB LE 3 Patients with non-sustained ventricular tachycardia episodes notified by remote monitoring in each of the three selected periods, according to demographic and clinical variables

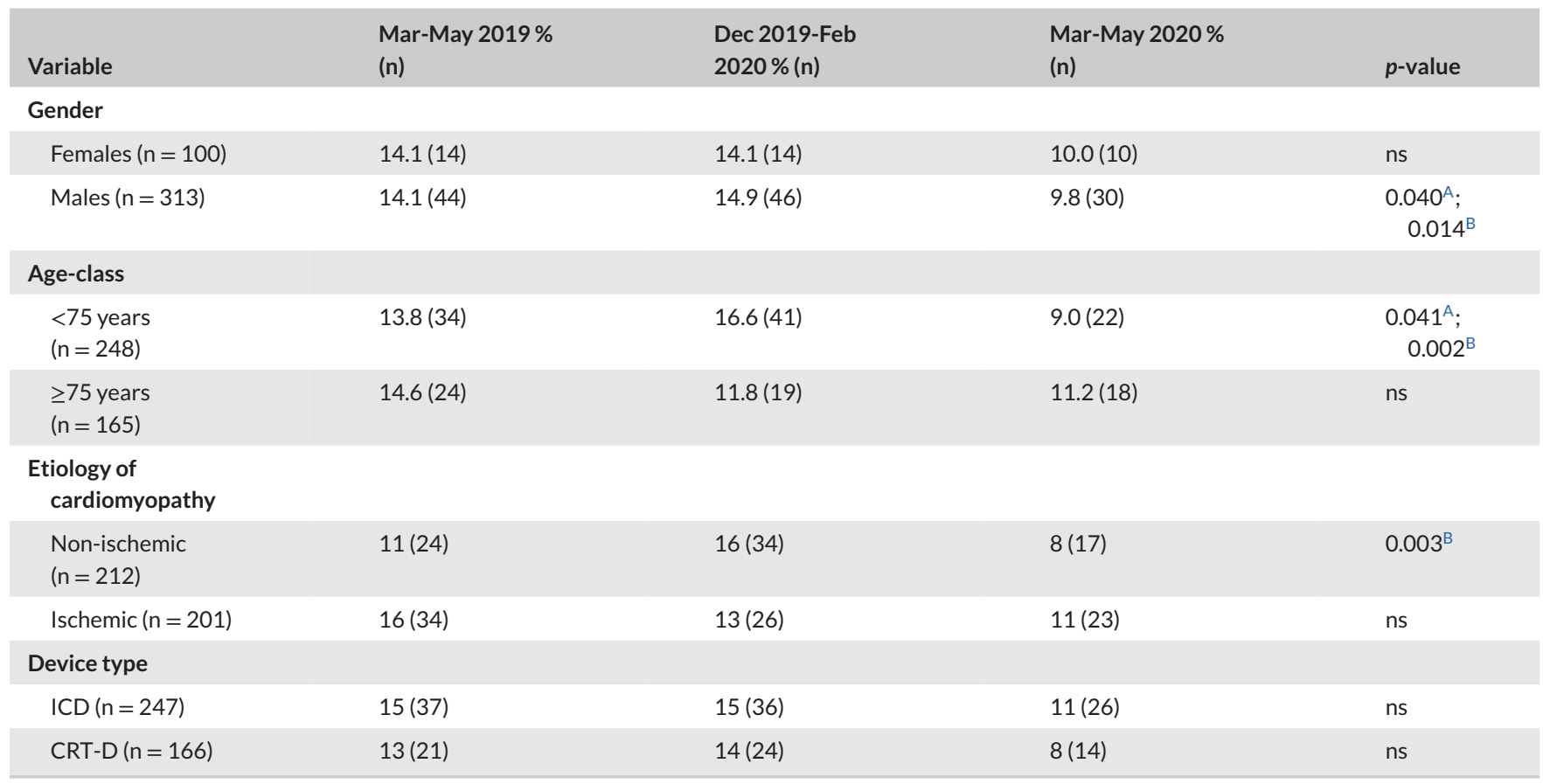

$\dagger$ McNemar's test:

A Mar-May 2020 versus Mar-May 2019;

B Mar-May 2020 versus Dec 2019-Feb 2020. All other $p$-values were not significant ( $>0.05$ ).

CRT-D, cardiac resynchronization therapy with defibrillator back-up; ICD, implantable cardioverter-defibrillator; Dec, December; Feb, February; Mar, March; ns, not significant.

It is assumed that the lockdown has negatively impacted lifestyles and mental health. Sedentary behavior, unhealthy diet, higher rate of smoking, greater alcohol intake, and less optimal medical therapy have been reported in Western countries that have adopted temporary lockdown strategies to counteract COVID-19 spread during the sharp rise in the pandemic. ${ }^{5}$ In addition, a negative psychological impact has been documented in socially marginalized subjects during COVID-19 quarantine. ${ }^{6}$ Fear of contagion or job loss with prolonged social isolation and economic concerns are common causes of mood disorders, mostly represented by traits or symptoms of depression and anxiety, especially in younger adults. Several reports have documented a causal relationship between acute mental stress and ventricular arrhythmias. $^{7,8}$ However, the literature is controversial regarding the existence of a clear relationship between depression or anxiety and the incidence of cardiac arrhythmias in ICD patients. ${ }^{9-14}$ Also, how these factors act as potential triggers for cardiac arrhythmias remains unclear. Likewise, evidence derived from population-wide major catastrophic events is inconsistent regarding the triggering effect of stress on ventricular arrhythmias in patients with ICD. ${ }^{15-17}$ While acknowledging that mental stressors might have been highly prevalent in our patients during the lockdown, we did not observe any increase in arrhythmic events or delivered ICD therapies.

Finally, limited opportunities to perform physical activity as a consequence of the mandatory in-home confinement have raised concerns for the maintenance of the well-established favorable effects of physi- cal activity on cardiovascular risk. ${ }^{18}$ Light-to-moderate exercise training programs in ICD recipients have resulted in similar benefits as those in other patients with cardiovascular disease. ${ }^{19-22}$ Accordingly, personalized programs for keeping physically active during the lockdown are strongly recommended, especially for older patients. ${ }^{23} \mathrm{We}$ recently analyzed data acquired by embedded accelerometer sensors as part of the rate-responsive pacing systems. In these ICD patients, whose movements were restricted as a consequence of the lockdown, a decrease of daily physical activity of $25 \%$ was observed. ${ }^{24}$ However, this reduction in physical activity did not translate into a higher prevalence of cardiac arrhythmias in our sample. We can speculate that a decline in physical activity in an already poorly trained ICD patient population might not have been of clinical significance. Moreover, a 10-week period may not have been long enough to result in a clinically significant loss of metabolic, cardiovascular and musculoskeletal conditioning.

A significant decrease in NSVT was observed for men, younger patients and those with ischemic cardiomyopathy. Since younger male patients represented the largest number of subjects still working in our sample, it is possible that during the stay-at-home order the forced rest has mitigated the job-related stress somewhat. A relationship between psychological stress and NSVTs has been demonstrated, especially in ischemic patients, likely through an increase in adrenergic tone. ${ }^{25}$ Additionally, recent data from an online social media-based survey of 135 individuals reported a significant increase of sleep duration by $11 \%$ 
during the stay-at-home order. ${ }^{26}$ Noteworthy, men showed a greater increase in the duration of sleep than women. Since sleep deprivation is an independent risk factor for cardiovascular disease, ${ }^{27}$ and improvement in sleep habits during the lockdown might have had a positive health impact on our patients.

Importantly, the only previous study on the topic, which included a cohort of 5963 ICD patients from 20 USA centers, followed over a 100day period during the pandemic, showed a significant decline in the risk of ventricular arrhythmias (VT or ventricular fibrillation) requiring device therapy. ${ }^{28}$ In contrast, we only found a non-significant reduction. The most likely explanation for the observed discrepancy lays in the shorter length of the follow-up and smaller sample size. O'Shea and Colleagues observed a progressive reduction of incident ventricular arrhythmias during a 14-week lockdown period: during the first week, 1923 (11.4\%) episodes were recorded, as compared to 482 in the final week. Since our follow-up lasted only 10 weeks, we may have missed a large amount of the reduction. This, combined with the substantially smaller sample of this study, as compared with the US one (414 vs. 5963 patients, respectively), may have determined the lack of significance of the decrease in either sustained VTs or appropriate device therapy (ATP or shock) for ventricular arrhythmias in our study.

This study has potential limitations. Firstly, our data were partly retrospective. Secondly, the short study period certainly represents a limitation, however the extension of the study period beyond the end of the national lockdown period would have introduced an unmeasured bias related to the possibility of moving within the country borders and the resumption of physical activity which may have impacted the arrhythmic burden in ICD patients. Thirdly, we did not systematically assess either the mental health or sleep patterns or other health outcomes thus any conclusion on the association between pandemicrelated psychological disorders or sleep habits and arrhythmic events remains merely speculative. Finally, the presence of possible confounding factors (e.g., individual training level, type of diet, alcohol intake) was not analyzed, thus making it difficult to draw definitive conclusions. Owing to these limitations, we are aware that our analysis remains descriptive in nature and should be considered hypothesisgenerating, opening the path for further studies and mechanistic research.

\section{5 | CONCLUSION}

At present, the impact of restrictive lockdown measures in response to COVID-19 on the arrhythmic event rate in patients with cardiac implantable electronic devices is largely unknown. We reported on the arrhythmic consequences of the total lockdown to contain the COVID-19 outbreak, in an urban cohort of patients with ICDs. The abrupt and enforced temporary in-home confinement, as mandated by the national lockdown in Italy, was not associated with increased risk of either cardiac arrhythmias or delivered device therapies.

\section{DISCLOSURE}

The authors have no conflicts to disclose for the content of this article and this research did not receive any specific grant from funding agencies in the public, commercial, or not-for-profit sectors.

\section{AUTHOR CONTRIBUTIONS}

Biagio Sassone: Study concept and research design, data interpretation, drafting article, approval of the final version of the article; Santo Virzì: Data collection, critical revision of the manuscript, approval of the final version of the article; Matteo Bettini: data collection, critical revision of the manuscript, approval of the final version of the article; Giovanni Pasanisi: Data collection, critical revision of the manuscript, approval of the final version of the article; Lamberto Manzoli: Statistics, critical revision of the manuscript, approval of the final version of the article; Jonathan Myers: Drafting article, critical revision of manuscript, approval of the final version of the article; Giovanni Grazzi: Data interpretation, drafting article, approval of the final version of the article; Daniele Muser: Data interpretation, critical revision of the manuscript, approval of the final version of the article

\section{ORCID}

Biagio Sassone MD (ID) https://orcid.org/0000-0002-9028-7169

Daniele Muser MD, PhD (DD https://orcid.org/0000-0003-4323-5988

\section{REFERENCES}

1. Wang D, Hu B, Hu C, et al. Clinical characteristics of 138 hospitalized patients with 2019 novel coronavirus infected pneumonia in Wuhan, China. JAMA. 2020;323:1061-1069.

2. Bhatla A, Mayers MM, Adusumalli S, et al. COVID-19 and cardiac arrhythmias. Heart Rhythm. 2020;17:1439-1444.

3. De Larochellière $\mathrm{H}$, Champagne J, Sarrazin JF, et al. Findings of remote monitoring of implantable cardioverter defibrillators during the COVID-19 pandemic. Pacing Clin Electrophysiol. 2020;43:13661372.

4. Haider N, Osman AY, Gadzekpo A, et al. Lockdown measures in response to COVID-19 in nine sub-Saharan African countries. BMJ Global Health. 2020;5:e003319.

5. Pollard MS, Tucker JS, Green HD. Changes in adult alcohol use and consequences during the COVID-19 pandemic in the US. JAMA Network Open. 2020;3:e2022942. https://doi.org/10.1001/jamanetworkopen. 2020.22942

6. Ettman CK, Abdalla SM, Cohen GH, Sampson L, Vivier PM, Galea S. Prevalence of depression symptoms in US adults before and during the COVID-19 pandemic. JAMA Netw Open. 2020;3:e 2019686.

7. Ziegelstein RC. Acute emotional stress and cardiac arrhythmias. JAMA. 2007;298:324-329.

8. Reich P, DeSilva RA, Lown B, Murawski BJ. Acute psychological disturbances preceding life-threatening ventricular arrhythmias. JAMA. 1981;246:233-235

9. Lampert R, Joska T, Burg MM, Batsford WP, McPherson CA, Jain D. Emotional and physical precipitants of ventricular arrhythmia. Circulation. 2002;106:1800-1805.

10. van den Broek KC, Nyklicek I, van der Voort PH, Alings M, Meijer A, Denollet J. Risk of ventricular arrhythmia after implantable 
defibrillator treatment in anxious type D patients. J Am Coll Cardiol. 2009;54:531-537.

11. Fu Y, Shen X, Huang W. Association between depression and risk of triggering ventricular arrhythmias: a meta-analysis. Int J Clin Pharmacol Ther. 2019;57:306-314.

12. Habibović M, Pedersen SS, van den Broek KC, et al. Anxiety and risk of ventricular arrhythmias or mortality in patients with an implantable cardioverter defibrillator. Psychosom Med. 2013;75:36-41.

13. Manstenbroek MH, Versteeg H, Jordaens L, Theuns DAMJ, Pedersen SS. Ventricular tachyarrhythmias and mortality in patients with an implantable cardioverter defibrillator: impact of depression in the MIDAS Cohort. Psychosom Med. 2014;76:58-65.

14. Andersen CM, Theuns DAMJ, Johansen JB, Pedersen SS. Anxiety, depression, ventricular arrhythmias and mortality in patients with an implantable cardioverter defibrillator: 7 years' follow-up of the MIDAS cohort. Gen Hosp Psychiatry. 2020;66:154-160.

15. Nishimoto Y, Firth BR, Kloner RA, Leor J, Lerman RD, Bhandari AK. The 1994 Northridge earthquake triggered shocks from implantable cardioverter-defibrillators. Circulation. 1995;92:2899.

16. Steinberg JS, Arshad A, Kowalski M, et al. Increased incidence of life-threatening ventricular arrhythmias in implantable defibrillator patients after the world trade center attack. J Am Coll Cardiol. 2004:44:1261-1264.

17. Chan C, Daly M, Melton I, Crozier I. Two major earthquakes in Christchurch were not associated with increased ventricular arrhythmias: analysis of implanted defibrillator diagnostics. PLoS ONE. 2019;14:e021652.

18. Lippi G, Henry BM. Sanchis-Gomar F. Physical inactivity and cardiovascular disease at the time of coronavirus disease 2019 (COVID-19). Eur J Prev Cardiol. 2020;27:906-908

19. Vanhees L, Kornaat M, Defoor J, et al. Effect of exercise training in patients with an implantable cardioverter defibrillator. Eur Heart J. 2004;25:1120-1126

20. Alswyan AH, Sauer Liberato AC, Dougherty CM. A systematic review of exercise training in patients with cardiac implantable devices. $J$ Cardiopulm Rehabil Prev. 2018:38:70-84.
21. Dougherty CM, Burr RL, Kudenchuk PJ, Glenny RW. Aerobic exercise effects on quality of life and psychological distress after an implantable cardioverter defibrillator. J Cardiopulm Rehabil Prev. 2020;40:94-101.

22. Steinhaus DA, Lubitz SA, Noseworthy PA, Kramer DB. Exercise interventions in patients with implantable cardioverter defibrillators and cardiac resynchronization therapy. J Cardiopulm Rehabil Prev. 2019;39:308-317.

23. Pavon DJ, Baeza AC, Lavie CJ. Physical exercise as therapy to fight against the mental and physical consequences of COVID-19 quarantine: special focus in older people. Prog Cardiovasc Dis. 2020;63:386388.

24. Sassone B, Mandini S, Grazzi G, Mazzoni G, Myers J, Pasanisi G. Impact of COVID-19 pandemic on physical activity in patients with implantable cardioverter-defibrillators. J Cardiopulm Rehabil Prev. 2020;40:285-286.

25. Brodsky MA, Sato DA, Iseri LT, Wolff LJ, Allen BJ. Ventricular tachycardia associated with psychological stress. The role of the sympathetic nervous system. JAMA. 1987;257:2064-2067.

26. Advani I, Gunge D, Banks S, et al. Is increased sleep responsible for reductions in myocardial infarction during the COVID-19 pandemic? Am J Cardiol. 2020; 131:127-140

27. Ayas NT, White DP, Manson JE, et al. A prospective study of sleep duration and coronary heart disease in women. Arch Intern Med. 2003;163:205-209.

28. O'Shea CJ, g Thomas, Middeldorp ME, et al. Ventricular arrhythmia burden during the coronavirus disease 2019 (COVID-19) pandemic. Euro Heart J. 2021;42:520-528.

How to cite this article: Sassone B, Virzì S, Bertini M, et al. Impact of the COVID-19 lockdown on the arrhythmic burden of patients with implantable cardioverter-defibrillators. Pacing Clin Electrophysiol. 2021;44:1033-1038.

https://doi.org/10.1111/pace.14280 